CLINICAL STUDY

\title{
Acylation-stimulating protein increases and correlates with increased progesterone levels during the luteal phase of the menstrual cycle
}

\author{
Jumana Saleh $^{1}$, Manal Al-Khanbashi ${ }^{1}$, Majida Al-Maarof ${ }^{2}$, Mohsin Al-Lawati $^{1}$, Syed G Rizvi ${ }^{3}$ \\ and Katherine Cianflone ${ }^{4}$ \\ Departments of ${ }^{1}$ Biochemistry, ${ }^{2}$ Obstetrics and Gynecology and ${ }^{3}$ Family Medicine and Public Health, Sultan Qaboos University, Muscat 123, Sultanate of \\ Oman and ${ }^{4}$ Centre de Recherche Hopital Laval, Laval University, Laval, Quebec G1V 4G5, Canada \\ (Correspondence should be addressed to J Saleh; Email: jumana@squ.edu.om)
}

\begin{abstract}
Objective: The menstrual cycle represents a continuous state of change in terms of female sex steroid environment. Progesterone is linked to increased fat storage while estrogen exerts anti-lipogenic effects. This study investigated variations in the potent lipogenic factor acylation-stimulating protein (ASP), and examined its association with hormonal and lipid profile alterations across the menstrual cycle.

Methods and design: Nineteen non-obese women with regular menstrual cycles were investigated in a longitudinal study during the follicular, ovulatory, and mid-luteal phases (ML) of the cycle. Fasting ASP, LH, FSH, progesterone, estradiol, insulin, lipid profile, and apoproteins were evaluated during different phases of the cycle.

Results: ASP levels changed significantly throughout the menstrual cycle (K-related Friedman test: $P=0.013)$. Interestingly, these changes coincide with variations in progesterone levels across the cycle as no significant change in the ASP levels was seen across the follicular phases of the cycle, followed by a significant increase in the ovulatory phase, which continued to elevate toward the ML. The ASP levels correlated positively with the progesterone levels normally elevated in the ML. No significant correlation was seen between ASP and estrogen or any other measured female hormone. Multiple regression analysis including all measured parameters and body mass index showed that progesterone was the only significant predictor of the ASP levels.

Conclusion: Our findings suggest that during the menstrual cycle of normal women, the ASP levels coincidentally fluctuate with the progesterone levels, possibly reflecting cooperation between them in fat storage enhancement.
\end{abstract}

European Journal of Endocrinology $160301-307$

\section{Introduction}

Acylation-stimulating protein (ASP) is an adipokine shown to affect lipid metabolism in humans and mice (1). It is a $8.9 \mathrm{kDa}$ autocrine hormone produced through the alternate complement pathway by the interaction of complement factor C3 with factors B and $\mathrm{D}$ (also called adipsin), which results in the formation of C3a-des-Arg, also called ASP. In vitro, ASP increases triglyceride synthesis and storage in adipocytes through the activation of diacylglycerol acyltransferase, the ratelimiting enzyme in triglyceride synthesis, and by stimulating glucose uptake $(2,3)$. The effect of ASP on fat storage is supported by in vivo data showing that ASP administration increases triglyceride clearance in mice, and that ASP-deficient $\left(\mathrm{C}^{-/-}\right)$mice exhibit delayed postprandial lipid clearance and reduced adipose tissue depots (4-7). In humans, ASP released from adipose tissue increased postprandially in coordination with triglyceride clearance $(8,9)$. The ASP levels decreased during fasting and after weight loss $(10,11)$, and increased in obesity (12) and dyslipidemic disorders $(13,14)$.

Differences in fat distribution and lipid profile exist between males and females of reproductive age. In females, changes in fat distribution coincide with the onset of ovarian production of estrogen and progesterone during puberty (15-19) and with cessation of hormone production during menopause (20-22). Marked changes in female lipid metabolism occur during pregnancy $(23,24)$ and in reproductive disorders accompanied with hormonal changes (25).

Obesity and weight gain are major clinical concerns for women of reproductive age. It is recognized that female hormone alterations are associated with enhanced subcutaneous fat storage in females. 
However, the factors that directly determine weight gain and fat retention in women are not fully understood. Insulin and ASP are the most potent fat storage hormones known. Insulin has been the focus of studies concerning lipid metabolism in women (24). Limited information is available about the association of female hormones with ASP. Previously, it was shown that the ASP levels in obese females were higher than those in obese males (12). A significant correlation was found between the ASP levels and body mass index (BMI) in women (26), a factor that may contribute to increased subcutaneous fat retention and resistance to weight loss in women. It was also postulated that ASP binds with higher affinity to subcutaneous fat cells freshly prepared, when derived from women than when derived from men, suggesting a role for ASP that may be more effective in females than in males (39). These observations require further investigations about associations of ASP with female hormones.

Abundant in vitro and in vivo evidence showed that progesterone is the female hormone mainly associated with increased fat storage and adipocyte differentiation (27-29). Estrogen, on the other hand, exhibits antilipolytic effects such as inhibition of lipoprotein lipase activity and reducing fat storage in adipocytes (30-32). The effects of menstrual cycle hormone changes on factors directly linked to lipid metabolism as insulin and adipokines such as leptin and adiponectin have been thoroughly investigated (31-38). Limited evidence is available considering sex hormone effects on the ASP levels or function.

In this study, we investigate the ASP levels and their possible correlation with female hormone changes, lipid profile, and the lipogenic factor insulin during all the phases of the menstrual cycle (follicular, ovulatory (OV), and luteal) of normal women.

This is the first study to investigate in vivo variations in the ASP levels in association with major hormonal alterations in healthy women.

\section{Materials and methods}

\section{Subjects}

Nineteen women aged between 20 and 24 years enrolled for this longitudinal study. All of them were single and had regular menstrual cycles of an average \pm s.E.M. $28.7 \pm 1.4$ days. They were non-obese, never pregnant and had not take oral contraceptives. All participants were non-smokers and non-alcohol consumers and were not receiving any medication. Women with menstrual abnormalities or abnormal female hormone levels that suggest anovulatory cycles were removed from the study. The subjects for this study come from a considerably homogeneous Omani population, which is characterized by large tribes of extended families with similar lifestyles.
The study was approved by the Sultan Qaboos University ethical committee. Informed consent forms were filled out by all the women participating in the study.

\section{Blood collection}

Fasting blood samples were collected by venipuncture at days $1,7,12,14$, and 21 . These times were chosen to represent different phases of an average '28-day' menstrual cycle. Days 1 and 7 marked the early follicular (EF) and mid-follicular (MF) phases respectively. Day 12 marked the late follicular (LF) phase (i.e. pre-ovulatory phase). Day 14 marked the OV phase, which marks the onset of the luteal phase. The midluteal phase (ML) was represented by day 21 . Female hormone levels of all the samples were measured to verify the phase at which the samples were taken.

\section{Analysis}

Fasting, venous blood samples were collected in plain tubes with no anti-coagulant for serum lipid and hormone measurements and EDTA tubes for ASP measurements. The EDTA samples were immediately put on ice and centrifuged. Serum samples were centrifuged after $20 \mathrm{~min}$. The serum and plasma were separated and stored at $-80{ }^{\circ} \mathrm{C}$ until analysis.

\section{Lipid profile measurements}

The samples were analyzed for the fasting lipid profile at different phases of the menstrual cycle including triglycerides (TG), total cholesterol (T-CHOL), lowdensity lipoprotein cholesterol (LDL-C), very low-density lipoprotein cholesterol (VLDL-C), and high-density lipoprotein cholesterol (HDL-C). Analysis was performed using automated Roche Integra 800 analyzer, Switzerland-Germany. APOA1 and APOB were measured on the Beckman Coulter IMMAGE 800 Immunochemistry System (Chaska, MN, USA) in the central routine laboratory of the university hospital.

\section{Hormone measurements}

Hormone levels including estrogen, progesterone, LH, FSH, and insulin were measured at different phases of the menstrual cycle using an automated clinical chemistry analyzer Beckman Coulter ACCESS 2 Immunoassay System analyzer in the central routine laboratory of the university hospital.

\section{ASP measurement}

Fasting ASP levels at the different phases of the menstrual cycle were measured using a sensitive ELISA assay described elsewhere (8). The human plasma EDTA samples and controls were pretreated 
with polyethylene glycol PEG 8000 to precipitate C3, thus preventing any artifactual generation of ASP, as described previously (8). The samples were centrifuged; the supernatant was separated and diluted. ASP was assayed immediately after dilution using an MAB as capture antibody and a polyclonal antibody as detecting antibody. The linear range of the standard curve was from 0.146 to $3.08 \mathrm{nM}$. Intra-assay coefficient of variation $(\mathrm{CV})$ for the ASP ELISA was $4 \%$ and interassay $\mathrm{CV}$ was $8 \%$.

\section{Statistical analysis}

Results were expressed as mean \pm s.E.M. of variables at each phase of the menstrual cycle. Within-group differences at different phases were assessed by oneway repeated-measures ANOVA (RM ANOVA), and for non-parametric data Friedman's K-related samples were used. Once it was determined that differences exist among the means, pairwise comparisons were performed by paired $T$-test to compare the means of variable levels at different phases. The Wilcoxon signed-rank test was performed for pairwise comparisons of variables with skewed distributions. Correlations between plasma the ASP levels and all measured variables at different phases of the cycle were examined by bivariate analysis using Pearson coefficients. Spearman correlation coefficients were used for the parameters with skewed distributions. Stepwise multiple linear regression analysis was performed to determine the factors that were associated significantly with variations in the ASP levels. Significance was set at $P<0.05$. Analysis was computer assisted using SPSS software.

\section{Results}

The women were non-obese with an average BMI \pm S.E.M. of $21.5 \pm 0.74 \mathrm{~kg} / \mathrm{m}^{2}$ and waist to hip ratio of $0.74 \pm 0.01$.

Fasting ASP, progesterone, estradiol, progesterone/estrogen ratio, and insulin levels are shown in Figs 1 and 2. LH, FSH, insulin, TG, T-CHOL, LDL-C, VLDL-C, HDL-C, APOA1, and APOB levels are shown in Table 1. All parameters were measured at the EF, MF, LF, OV, and ML phases.

\section{PIasma ASP and insulin level changes during the menstrual cycle}

Figure 2A showed that fasting ASP levels changed significantly during the different phases of the menstrual cycle (K-related Friedman analysis, $P=0.013$ ). At the onset of menses on day 1 (EF phase), the ASP levels were $(18.7 \pm 1.9 \mathrm{nM})$. No significant change was seen in the MF $(17.3 \pm 1.8 \mathrm{nM})$ and $\mathrm{LF}(14.5 \pm 1.5 \mathrm{nM})$ phases as determined by Wilcoxon pairwise comparisons. The significant increase occurred in the OV phase
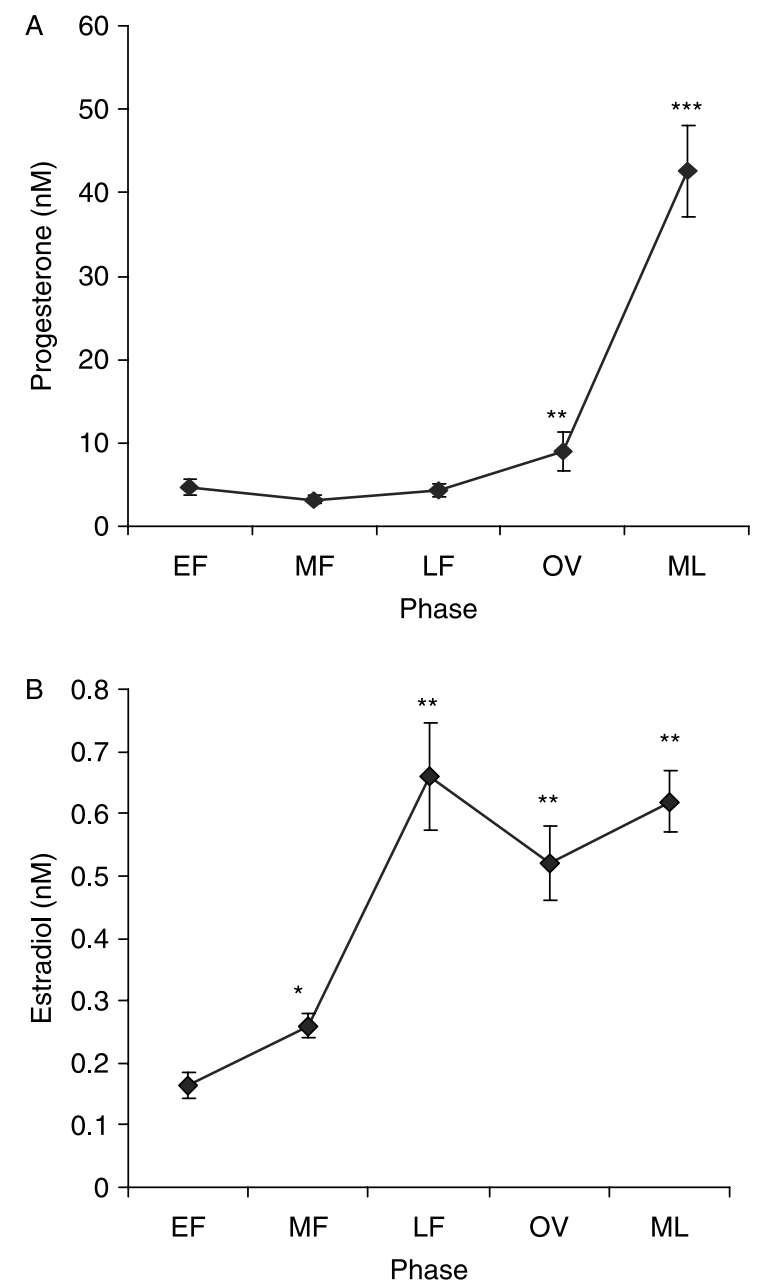

Figure 1 (A) Progesterone and (B) estradiol levels at the early follicular (EF), mid-follicular (MF), late follicular (LF), ovulatory (OV), and mid-luteal (ML) phases. Data at each phase is shown as the mean \pm S.E.M. $(n=19)$. Repeated-measures ANOVA: $P<0.0001$. Pairwise comparisons of the means were made by paired sample $T$-test. The Wilcoxon signed-rank test was used when the values of progesterone were skewed in the EF and OV phases and estrogen in the EF phase. Progesterone: ${ }^{* * *} P<0.001$ : ML phase higher than all phases. ${ }^{\star \star} P<0.05$ : OV phase higher than the EF phase.

Estradiol: ${ }^{* *} P<0.001$ in ML, OV, and LF higher than EF and MF phases. ${ }^{\star} P<0.02 \mathrm{MF}$ higher than the EF phase.

$(18.6 \pm 2.3 \mathrm{nM} ; P<0.05)$ in coordination with the significant increase in the progesterone levels at that stage followed by a twofold increase during the ML phase $(29.6 \pm 6.6 \mathrm{nM})$ compared with the LF phase $(P<0.02)$. The luteal increase in plasma ASP levels coincided with the increase in the progesterone levels and the progesterone/estrogen ratio (P: E) in the luteal (ML) phase Fig. 2B.

By contrast, fasting insulin levels did not show significant change during the cycle (RM ANOVA, $P=$ NS) and pairwise comparisons of insulin levels between different phases of the cycle were all non-significant 

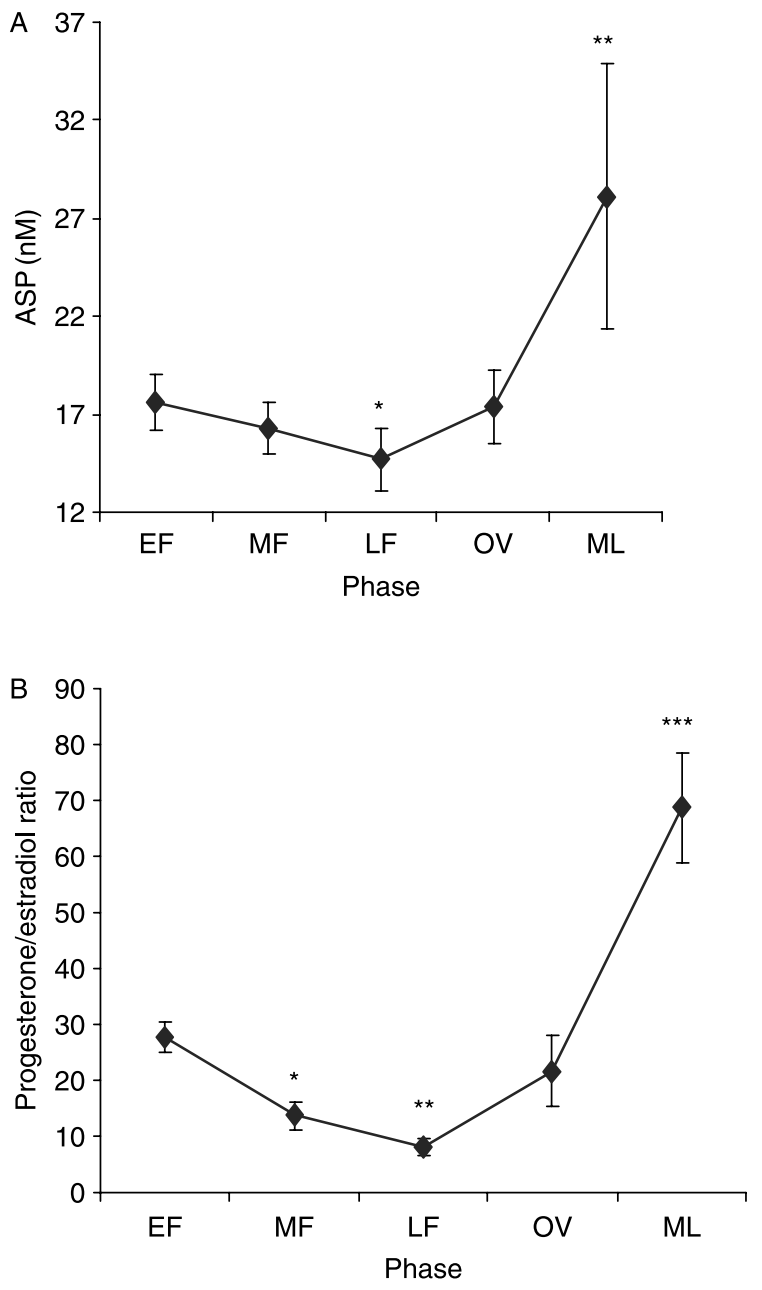

Figure 2 (A) ASP levels at the early follicular (EF), mid-follicular $(\mathrm{MF})$, late follicular (LF), ovulatory (OV), and mid-luteal (ML) phases. Data at each point (phase) is shown as mean \pm S.E.M. $(n=19)$. Pairwise comparisons of the means were made by paired sample $T$-test. The Wilcoxon signed-rank test was used when ASP levels were skewed in the ML phase. ${ }^{\star \star} P<0.02$ : ML higher than LF and MF phases. ${ }^{*} P<0.05$ : OV higher than LF phase. (B)

Corresponding levels of progesterone/estrogen ratio in the different phases are shown. ${ }^{\star * \star} P<0.001: \mathrm{ML}$ phase higher than all phases. ${ }^{\star *} P<0.05$ : LF compared with EF and $P<0.001$ compared with the MF phase, ${ }^{\star} P<0.001$ : MF compared with EF phase.

(Fig. 3). This is also reflected by the glucose levels that did not change significantly during the cycle (results not shown).

\section{Correlation of ASP with hormones and lipids during the $M L$}

Increased ASP levels in the ML phase showed a significant positive correlation with the progesterone levels and with the progesterone/estrogen ratio Table 2 . ASP also positively correlated with BMI (Table 2). A multiple regression model was set in order to determine the factors that predicted the ASP levels in the ML phase. Plasma ASP was set as the dependent variable. Hormone, lipid, and subject characteristics were entered into the model as predictors. These included progesterone, estradiol, LH, FSH, insulin, TG, LDL-C, HDL-C, VLDL-C, apoproteins, BMI, and age. The results showed that progesterone significantly associated with the ASP levels $(\beta=0.516, P=0.028)$ and entered this model as the only significant predictor. BMI was excluded as a non-significant predictor of the ASP levels when included in the multiple regression model. Progesterone levels significantly accounted for $22 \%$ variation in fasting (as determined by adjusted $R^{2}$ ). All other measured hormones, lipid parameters, and age were excluded from the model as non-significant. This correlation was found in the ML phase. No correlation was found between the ASP levels and the corresponding hormone and lipid parameters in other phases of the menstrual cycle.

By contrast, although insulin levels in the ML phase correlated positively with BMI $(r=0.44, P<0.05)$, it showed no significant correlation with the levels of progesterone, estrogen, or progesterone/estrogen ratio throughout the menstrual cycle phases.

\section{Discussion}

In this study, we showed expected reproductive hormonal alterations during the different phases of an average '28-day' menstrual cycle.

The key finding in this study was that the ASP levels changed significantly across the phases of the menstrual cycle showing a similar pattern of change as the progesterone levels and progesterone/estrogen ratio. The ASP levels were low across the follicular phases and significantly increased in the OV phase, which marks the onset of the luteal phase, followed by a marked (twofold) increase in the ML phase. Importantly, this increase in the ASP levels correlated positively with the normally elevated progesterone levels in the ML, whereas no significant association of ASP was seen with the estrogen levels or other measured reproductive hormones. The correlation of the ASP levels with the progesterone/estrogen ratio was similar to the correlation of ASP with the progesterone levels alone, further indicating that the estrogen levels had no association with the plasma ASP levels. The ASP levels correlated with the BMI of the females in this study, which is consistent with the previous findings in Omani women (26); however, a stronger correlation was found with the progesterone levels. Multiple regression analysis showed that progesterone was the only significant predictor of the ASP levels and excluded BMI as nonsignificant from the regression model. This may be due to the fact that all females included in this study were non-obese, highlighting the association between the ASP and progesterone levels independent of weight 
Table 1 Hormone and lipid profile levels shown at different phases of the menstrual cycle of healthy women.

\begin{tabular}{llllll}
\hline Variable $(n=19)$ & EF & MF & LF & OV & ML \\
\hline Progesterone & $4.7 \pm 1.1$ & $3.2 \pm 0.55$ & $4.4 \pm 0.85$ & $8.8 \pm 2.4$ & $42.6 \pm 5.9$ \\
Estrogen & $0.17 \pm 0.02$ & $0.26 \pm 0.03$ & $0.66 \pm 0.09$ & $0.50 \pm 0.06$ & $0.60 \pm 0.05$ \\
Progesterone/estrogen & $27.6 \pm 2.6$ & $13.7 \pm 2.5$ & $8.2 \pm 1.6$ & $21.7 \pm 6.4$ & $68.7 \pm 9.7$ \\
LH (IU/l) & $4.0 \pm 0.4$ & $6.0 \pm 0.5$ & $20.2 \pm 4.6^{1}$ & $23.9 \pm 5.3^{1}$ & $4.5 \pm 0.69$ \\
FSH (IU/l) & $7.0 \pm 0.67$ & $7.1 \pm 0.4$ & $7.3 \pm 0.79$ & $9.2 \pm 0.73^{2}$ & $3.5 \pm 0.35$ \\
TG (mM) & $0.85 \pm 0.05$ & $0.85 \pm 0.07$ & $0.76 \pm 0.06^{3}$ & $0.68 \pm 0.04^{4}$ & $0.75 \pm 0.07$ \\
T-CHOL (mM) & $5.2 \pm 0.16$ & $5.2 \pm 0.15$ & $4.9 \pm 0.18$ & $4.97 \pm 0.18$ & $5.1 \pm 0.13$ \\
LDL-C (mM) & $3.3 \pm 0.18$ & $3.3 \pm 0.18$ & $3.0 \pm 0.18^{5}$ & $3.15 \pm 0.17$ & $2.98 \pm 0.17^{5}$ \\
VLDL-C (mM) & $0.38 \pm 0.02$ & $0.38 \pm 0.03$ & $0.34 \pm 0.03$ & $0.31 \pm 0.02^{6}$ & $0.33 \pm 0.03$ \\
HDL-C (mM) & $1.7 \pm 0.08$ & $1.8 \pm 0.09$ & $1.8 \pm 0.09$ & $1.8 \pm 0.11$ & $1.87 \pm 0.1$ \\
APOAl (g/l) & $1.56 \pm 0.06$ & $1.6 \pm 0.07$ & $1.57 \pm 0.07$ & $1.59 \pm 0.07$ & $1.58 \pm 0.05$ \\
APOB (g/l) & $0.94 \pm 0.04$ & $0.9 \pm 0.04$ & $0.80 \pm 0.03^{7}$ & $0.88 \pm 0.03$ & $0.81 \pm 0.03^{8}$ \\
\hline
\end{tabular}

Results are shown as mean \pm s.E.M. LH, luteinizing hormone; FSH, follicular-stimulating hormone; TG, triglycerides; LDL, low-density lipoprotein; VLDL, very low-density lipoprotein; HDL, high-density lipoprotein; T-CHOL, total cholesterol; APOA, apoprotein A; APOB, apoprotein B; GLU, glucose; EF, early follicular; MF, mid-follicular; LF, late follicular; OV, ovulatory and ML, mid-luteal. Significance is indicated by superscript numbers as shown below. Note: Significant differences across the different points of the cycle for progesterone, estrogen, and progesterone/estrogen ratio are shown in Figs 1 and 2 . LH: ${ }^{1} \mathrm{OV}$ and ${ }^{1} \mathrm{LF}$ higher than $\mathrm{EF}, \mathrm{MF}$, and $\mathrm{ML}$ phases $(P<0.005)$. $\mathrm{FSH}:{ }^{2} \mathrm{OV}$ higher than $\mathrm{EF}, \mathrm{MF}(P<0.02)$, and $\mathrm{ML}$ phases $(P<0.001)$. TG: ${ }^{3} \mathrm{LF}$ lower than $\mathrm{MF}(P<0.05) .{ }^{4} \mathrm{OV}$ lower than EF $(P<0.01)$ and MF phases $(P<0.05)$. LDL-C: ${ }^{5} \mathrm{LF}$ and ${ }^{5} \mathrm{ML}$ lower than EF $(P<0.02)$ and MF phases $(P \leq 0.01)$. VLDL-C: ${ }^{6} \mathrm{OV}$ lower than EF phase $(P<0.05)$. APOB: ${ }^{7} \mathrm{LF}$ lower than EF and MF phases $(P<0.05) .{ }^{8} \mathrm{ML}$ lower than EF and MF phases $(P<0.01)$.

gain. The rest of the female hormones, insulin, and lipid parameters measured in the study were also excluded as non-significant. Although insulin (a major fat storage factor) correlated positively with BMI in the ML, it did not show any significant changes during the cycle and did not correlate with progesterone or estradiol levels. This agrees with several findings in the literature where no significant changes in insulin levels were seen across the cycle in healthy females $(37,38)$.

Recent studies support our findings of increased ASP levels in the luteal phase of the menstrual cycle. These studies, although not in plasma, showed that $\mathrm{C} 3$ and factor B (precursors of ASP) are produced in the human endometrium in a cycle-specific manner. It was found that luteal phase endometrium synthesizes complement

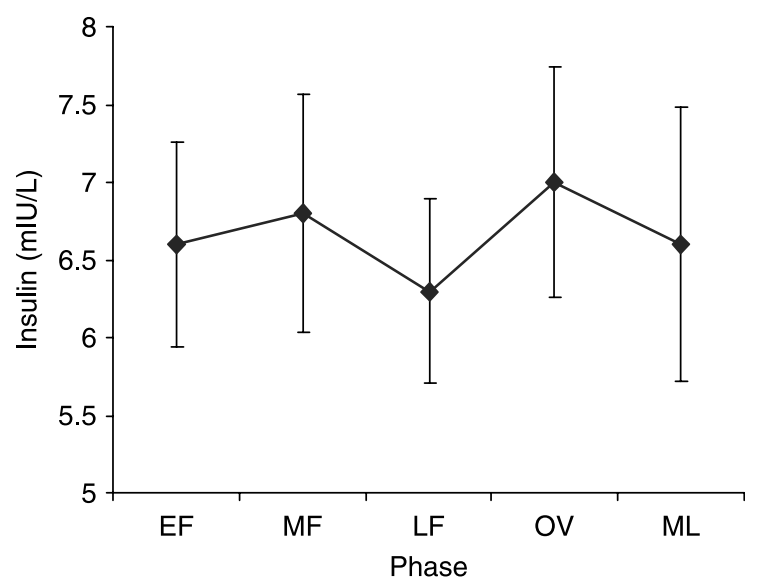

Figure 3 Insulin levels at different phases of the menstrual cycle. $\mathrm{EF}$, early follicular; MF, mid-follicular; LF, late follicular; OV, ovulatory; ML, mid-luteal. Data at each point (phase) is shown as mean \pm S.E.M. $(n=19)$. Pairwise comparisons of the means were made by paired sample $T$-test. No significant differences were found between any of the means in the different phases of the cycle.
C3 de novo, whereas proliferative endometrium produces little or no C3. Likewise, factor B, which is critical to the activation of the complement alternative pathway, 'which leads to ASP production', has been shown to be present only in the luteal phase endometrium and not in the follicular phase. Also, factor B was found to be synthesized in the endometrial cells of patients treated with exogenous progesterone therapy. Therefore, these precursors are found in the presence of high progesterone/estrogen ratio characterizing the luteal phase and not in the follicular phase, which is marked with low progesterone and estrogen levels $(40,41)$.

Although progesterone is suggested to exert lipogenic effects in females, controversy still exists as to whether it mediates these effects directly or indirectly. Some studies suggested direct anabolic effects through specific transcription factors, whereas others suggested that it

Table 2 Bivariate correlation between acylation-stimulating protein (ASP) levels in the mid-luteal (ML) phase with anthropometric, hormone, and lipid profile levels.

\begin{tabular}{lll}
\hline $\boldsymbol{n}=\mathbf{1 9}$ & ASP & Sig (two-tailed) \\
\hline BMI & $r=0.47^{*}$ & $P=0.04$ \\
Age & $r=-0.005$ & $P=0.98$ \\
Progesterone & $r=0.56^{*}$ & $P=0.012$ \\
Estradiol & $r=0.261$ & $P=0.25$ \\
P/E ratio & $r=0.60^{*}$ & $P=0.007$ \\
LH & $r=-0.023$ & $P=0.92$ \\
FSH & $r=-0.358$ & $P=0.11$ \\
Insulin & $r=0.038$ & $P=0.87$ \\
TG & $r=-0.88$ & $P=0.71$ \\
T-CHOL & $r=0.27$ & $P=0.26$ \\
VLDL-C & $r=-0.067$ & $P=0.77$ \\
LDL-C & $r=0.32$ & $P=0.16$ \\
HDL-C & $r=0.10$ & $P=0.66$ \\
APOB & $r=0.421$ & $P=0.58$ \\
APOA1 & $r=-0.03$ & $P=0.88$ \\
\hline
\end{tabular}

${ }^{\star} P$, Spearman correlation significant, two-tailed; Sig, significance level. 
enhanced insulin action $(27-30,42)$. This study is of particular interest as it is the first study in vivo to establish a link between progesterone, known for its lipogenic effects in females, and ASP, which is a potent fat storage factor. Showing progesterone as the only significant predictor of the ASP levels in non-obese women may suggest that this hormone may have a significant role in enhancing ASP production in females. This, however, does not exclude the possibility that high levels of ASP may also reflect ASP resistance as in hyperlipidemia during pregnancy or reproductive disorders $(26,43)$.

No evident correlation was seen between the ASP levels and the lipid profile of these healthy women, most probably because they are in the fasting state where lipid levels are normally low. Importantly, however, we found that lipid levels generally decrease during the second half of the cycle in agreement with the previous studies that suggest anti-atherogenic profiles in the females of reproductive age (44-46). Table 1 shows that during the ovulatory phase, which marks the beginning of the luteal phase, TGs were at their lowest levels showing $20 \%$ decrease $(P<0.01)$ compared with the early and mid-follicular phases. Moreover, VLDL-C levels, which represent the major circulating TG-rich lipoprotein during fasting, showed a $18.4 \%$ decrease at the ovulatory phase $(P<0.05)$ compared with the early and mid-follicular phases. This decrease in plasma TG parameters suggests enhanced TG clearance, hence increased fat storage notably coinciding with the initial increase in the ASP levels. We therefore speculate that TG clearance may be most evident at optimal ASP levels during the ovulatory phase, and continues toward the ML, although, to a lesser extent as ASP exceeds its optimal functional levels. Interestingly, these effects were seen in coordination with the significant increase in the progesterone levels, which was the main predictor of the ASP levels in this study. This close coordination between these metabolic parameters and TG clearance may suggest a role for progesterone in enhancing ASP production and therefore fat storage in women. However, this association does not prove causality and more in vitro and animal studies are warranted to confirm this hypothesis.

This is the first study showing variations in the ASP levels during the menstrual cycle and its correlation with increased progesterone levels, which may contribute to further understanding of the mechanism of ASP regulation regarding fat storage and distribution in women.

These findings also lend importance to the fact that menstrual cycle phases should be taken into consideration when collecting blood samples to perform studies on ASP and lipid levels in women of reproductive age.

\section{Declaration of interest}

The authors declare that there is no conflict of interest that could be perceived as prejudicing the impartiality of the research reported.

\section{Funding}

This work was supported by a project grant (IG/MED/BIOC/06/04) from Sultan Qaboos University.

\section{Acknowledgements}

We express our sincere gratitude to all the women who participated in the study. Prof. Katherine Cianflone is supported by a senior Canada Research Chair in Adipose Tissue.

\section{References}

1 Sniderman AD, Maslowska M \& Cianflone K. Of mice and men (and women) and the acylation-stimulating protein pathway. Current Opinion in Lipidology 200011 291-296.

2 Cianflone K, Roncari DAK, Maslowska M, Baldo A, Forden J \& Sniderman AD. The adipsin/acylation stimulating protein system in human adipocytes: regulation of triacylglycerol synthesis. Biochemistry 199433 9489-9495.

3 Cianflone K \& Maslowska M. Differentiation induced production of ASP in human adipocytes. European Journal of Clinical Investigation $199525817-825$.

4 Murray I, Havel PJ, Sniderman AD \& Cianflone K. Reduced body weight, adipose tissue, and leptin levels despite increased energy intake in female mice lacking acylation-stimulating protein. Endocrinology 2000141 1041-1049.

5 Murray I, Sniderman AD, Havel PJ \& Cianflone K. Acylation stimulating protein (ASP) deficiency alters postprandial and adipose tissue metabolism in male mice. Journal of Biological Chemistry 1999274 36219-36225.

6 Murray IA, Sniderman AD \& Cianflone K. Enhanced triglyceride clearance with intraperitoneal human acylation stimulating protein (ASP) in C57Bl/6 mice. American Journal of Physiology. Endocrinology and Metabolism 1999277 E474-E480.

7 Saleh J, Blevins JE, Havel PJ, Barrett JA, Gietzen DW \& Cianflone K. Acylation stimulating protein (ASP) acute effects on postprandial lipemia and food intake in rodents. International Journal of Obesity and Related Metabolic Disorders 200125 705-713.

8 Saleh J, Summers LKM, Cianflone K, Fielding BA, Sniderman AD \& Frayn KN. Coordinated release of acylation stimulating protein (ASP) and triacylglycerol clearance by human adipose tissue in vivo in the postprandial period. Journal of Lipid Research 199839 884-891.

9 Cianflone K, Zakarian R, Couillard C, Delplanque B, Despres JP \& Sniderman A. Fasting acylation-stimulating protein is predictive of postprandial triglyceride clearance. Journal of Lipid Research 2004 45 124-131.

10 Cianflone K, Sniderman AD, Kalant D, Marliss EB \& Gougeon R. Response of plasma ASP to a prolonged fast. International Journal of Obesity 199519 604-609.

11 Sniderman AD, Cianflone K \& Eckel RH. Levels of acylation stimulating protein in obese women before and after moderate weight loss. International Journal of Obesity 199115 333-336.

12 Maslowska M, Vu H, Phelis S, Sniderman AD, Rhode BM, Blank D \& Cianflone K. Plasma acylation stimulating protein, adipsin and lipids in non-obese and obese populations. European Journal of Clinical Investigation $199929679-686$.

13 Cianflone K, Zhang XJ, Genest J Jr \& Sniderman AD. Plasma acylation stimulating protein in coronary artery disease. Arteriosclerosis, Thrombosis, and Vascular Biology 199717 1239-1244.

14 Cianflone KM, Maslowska MH \& Sniderman AD. Impaired response of fibroblasts from patients with hyperapobetalipoproteinemia to acylation-stimulating protein. Journal of Clinical Investigation 199085 722-730.

15 de Ridder CM, Bruning PF, Zonderland ML, Thijssen JH, Bonfrer JM, Blankenstein MA, Huisveld IA \& Erich WB. Body fat 
mass, body fat distribution, and plasma hormones in early puberty in females. Journal of Clinical Endocrinology and Metabolism 1990 70 888-893.

16 Chumlea WC, Roche AF, Siervogel RM, Knittle JL \& Webb P. Adipocytes and adiposity in adults. American Journal of Clinical Nutrition 198134 1798-1803.

17 Chumlea WC, Knittle JL, Roche AF, Siervogel RM \& Webb P. Size and number of adipocytes and measures of body fat in boys and girls 10 to 18 years of age. American Journal of Clinical Nutrition $1981341791-1797$.

18 Ferin M, Jewelewicz R \& Warren M. The menstrual cycle: physiology, reproductive disorders, and infertility. New England Journal of Medicine 19943301097.

19 Landgren BM, Unden AL \& Diczfalusy E. Hormonal profile of the cycle in 68 normally menstruating women. Acta Endocrinologica $19809489-98$.

20 Ley CJ, Lees B \& Stevenson JC. Sex- and menopause-associated changes in body-fat distribution. American Journal of Clinical Nutrition $1992 \mathbf{5 5}$ 950-954.

21 Tchernof A, Poehlman ET \& Després JP. Body fat distribution, the menopause transition, and hormone replacement therapy. Diabetes E Metabolism 200026 12-20.

22 Poehlman E, Toth MJ \& Gardner AW. Changes in energy balance and body composition at menopause: a controlled longitudinal study. Annals of Internal Medicine 1995123 673-675.

23 Norton M, Buchanan TA \& Kitzmiller J. Endocrine pancreas and maternal metabolism. In Maternal-Fetal Endocrinology, pp 48-77. Eds D Tulchinsky \& A Brian Little, Philadelphia, PA: W.B Saunders Company, 1994.

24 Blackburn ST \& Loper DL. Carbohydrate, fat, and protein metabolism. In Maternal, Fetal and Neonatal Physiology, A Clinical Perspective, pp 583-613. Ed. T Eoyang, Philadelphia, PA: W.B Saunders Company, 1992.

25 Berneis K, Rizzo M, Lazzarini V, Fruzzetti F \& Carmina E. Atherogenic lipoprotein phenotype and low-density lipoproteins size and subclasses in women with polycystic ovary syndrome. Journal of Clinical Endocrinology and Metabolism 200792 186-189.

26 Saleh J, Cianflone K, Chaudhary T, Al-Riyami H, Al-Abri A \& Bayoumi R. Increased plasma acylation-stimulating protein correlates with hyperlipidemia at late gestation. Obesity 2007 15 646-652.

27 Lobo MJ, Remesar X \& Alemany M. Effect of chronic intravenous injection of steroid hormones on body weight and composition of female rats. Biochemistry and Molecular Biology International 1993 29 349-358.

28 Mendes AM, Madon RJ \& Flint DJ. Effects of cortisol and progesterone on insulin binding and lipogenesis in adipocytes from normal and diabetic rats. Journal of Endocrinology $1985 \mathbf{1 0 6}$ 225-231.

29 Kalkhoff RK. Metabolic effects of progesterone. American Journal of Obstetrics and Gynecology 1982142 735-738.

30 Homma H, Kurachi H, Nishio Y, Takeda T, Yamamoto T, Adachi K, Morishige K-I, Ohmichi M, Matsuzawa Y \& Murata Y. Estrogen suppresses transcription of lipoprotein lipase gene. Existence of a unique estrogen response element on the lipoprotein lipase promoter. Journal of Biological Chemistry $2000 \mathbf{2 7 5}$ 11404-11411.

31 Cooke PS \& Naaz A. Role of estrogens in adipocyte development and function. Experimental Biology and Medicine 2004229 1127-1135.

32 Cucinelli F, Paparella P, Soranna L, Barini A, Cinque B, Mancuso S \& Lanzone A. Differential effect of transdermal estrogen plus progestagen replacement therapy on insulin metabolism in postmenopausal women: relation to their insulinemic secretion. European Journal of Endocrinology 1999140 215-223.

33 Hardie L, Trayhurn P, Abramovich D \& Fowler P. Circulating leptin in women: a longitudinal study in the menstrual cycle and during pregnancy. Clinical Endocrinology 199747 101-106.

34 Cella F, Giordano G \& Cordera R. Serum leptin concentrations during the menstrual cycle in normal-weight women: effects of an oral triphasic estrogen-progestin medication. European Journal of Endocrinology $2000 \mathbf{1 4 2} 174-178$.

35 Ludwig M, Klein HH, Diedrich K \& Ortmann O. Serum leptin concentrations throughout the menstrual cycle. Archives of Gynecology and Obstetrics 2000263 99-101.

36 Kleiblová P, Springer D \& Haluzík M. The influence of hormonal changes during menstrual cycle on serum adiponectin concentrations in healthy women. Physiological Research $2006 \mathbf{5 5}$ 661-666.

37 Juul A, Scheike T, Pedersen AT, Main KM, Andersson AM, Pedersen LM \& Skakkebaek NE. Changes in serum concentrations of growth hormone, insulin, insulin-like growth factor and insulin-like growth factor-binding proteins 1 and 3 and urinary growth hormone excretion during the menstrual cycle. Human Reproduction 199712 2123-2128.

38 Riad-Gabriel M, Jinagouda SD, Sharma A, Boyadjian R \& Saad M. Changes in plasma leptin during the menstrual cycle. European Journal of Endocrinology $1998139528-531$.

39 Saleh J, Christou N \& Cianflone K. Regional specificity of ASP binding in human adipose tissue. American Journal of Physiology 1999276 E815-E821.

40 Hasty LA, Lambris JD, Lessey BA, Pruksananonda K \& Lyttle CR. Hormonal regulation of complement components and receptors throughout the menstrual cycle. American Journal of Obstetrics and Gynecology 1994170 168-175.

41 Hasty LA, Brockman WW, Lambris JD \& Lyttle CR. Hormonal regulation of complement factor $\mathrm{B}$ in human endometrium. American Journal of Reproductive Immunology 199330 363-367.

42 Lacasa D, Le Liepvre X, Ferre P \& Dugail I. Progesterone stimulates adipocyte determination and differentiation $1 /$ sterol regulatory element-binding protein $1 \mathrm{c}$ gene expression. Potential mechanism for the lipogenic effect of progesterone in adipose tissue. Journal of Biological Chemistry 2001276 11512-11516.

43 Wu Y, Zhang J, Wen Y, Wang H, Zhang M \& Cianflone K. Increased acylation-stimulating protein, C-reactive protein, and lipid levels in young women with polycystic ovary syndrome. Fertility and Sterility, 2008 DOI: 10.1016/j.fertnstert.2007.11.031.

44 Barnett JB, Woods MN, Lamon-Fava S, Schaefer EJ, McNamara JR, Spiegelman D, Hertzmark E, Goldin B, Longcope C \& Gorbach SL. Plasma lipid and lipoprotein levels during the follicular and luteal phases of the menstrual cycle. Journal of Clinical Endocrinology and Metabolism $200489776-782$.

45 Woods M, Schaefer EJ, Morrill A, Goldin BR, Longcope C, Dwyer JD \& Gorbach SL. Effect of menstrual cycle phase on plasma lipids. Journal of Clinical Endocrinology and Metabolism 198765 321-323.

46 Muesing RA, Forman MR, Graubard BI, Beecher GR, Lanza E, McAdam PA, Campbell WS \& Olson BR. Cyclic changes in lipoprotein and apolipoprotein levels during the menstrual cycle in healthy premenopausal women on a controlled diet. Journal of Clinical Endocrinology and Metabolism 199681 3599-3603.

Received 10 October 2008

Accepted 11 November 2008 